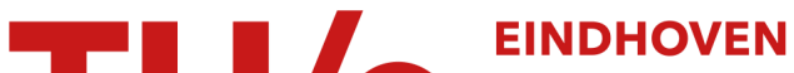 \\ UNIVERSITY OF \\ TECHNOLOGY
}

\section{Extended modeling for time-encoding converters}

Citation for published version (APA):

Roermund, van, A. H. M., Arfaei Malekzadeh, F., Sarkeshi, M., \& Mahmoudi, R. (2010). Extended modeling for time-encoding converters. In Proceeding of IEEE International Symposium on Circuits and Systems (ISCAS), 30 May - 2 June 2010, Paris, France (pp. 1077-1080) https://doi.org/10.1109/ISCAS.2010.5537345

DOI:

10.1109/ISCAS.2010.5537345

Document status and date:

Published: 01/01/2010

\section{Document Version:}

Publisher's PDF, also known as Version of Record (includes final page, issue and volume numbers)

\section{Please check the document version of this publication:}

- A submitted manuscript is the version of the article upon submission and before peer-review. There can be important differences between the submitted version and the official published version of record. People interested in the research are advised to contact the author for the final version of the publication, or visit the $\mathrm{DOI}$ to the publisher's website.

- The final author version and the galley proof are versions of the publication after peer review.

- The final published version features the final layout of the paper including the volume, issue and page numbers.

Link to publication

\section{General rights}

Copyright and moral rights for the publications made accessible in the public portal are retained by the authors and/or other copyright owners and it is a condition of accessing publications that users recognise and abide by the legal requirements associated with these rights.

- Users may download and print one copy of any publication from the public portal for the purpose of private study or research.

- You may not further distribute the material or use it for any profit-making activity or commercial gain

- You may freely distribute the URL identifying the publication in the public portal.

If the publication is distributed under the terms of Article 25fa of the Dutch Copyright Act, indicated by the "Taverne" license above, please follow below link for the End User Agreement:

www.tue.nl/taverne

Take down policy

If you believe that this document breaches copyright please contact us at:

openaccess@tue.nl

providing details and we will investigate your claim. 


\title{
Extended Modelling for Time-Encoding Converters
}

\author{
Arthur van Roermund, Foad Arfaei Malekzadeh, Mehdi Sarkeshi, Reza Mahmoudi \\ Mixed-signal Microelectronics group \\ Eindhoven University of Technology \\ Eindhoven, The Netherlands
}

\begin{abstract}
Amplitude-quantized time-encoding is beneficial in terms of Shannon capacity, a.o. for data converters. In case of asynchronous, it can be used too for power converters and amplifiers, with extra advantages in terms of power efficiency, power control, in-band quantizer distortion, and absence of both clock-induced noise and power dissipation. This paper fills in the lack of analysis and synthesis tools, for random inputs, with special focus on not yet addressed spectral-domain metrics. The strongly-non-linear quantizer function is translated to a weaklynon-linear one; Hermite expansion is used to achieve an analytical expression for the Shannon-defined SNR; and a 3-step insightful and fast synthesis approach, including a tradeoff between SNR and efficiency, is proposed. The approach is universal and can also be applied to synchronous converters.
\end{abstract}

\section{INTRODUCTION}

A time-encoder (TE) transforms amplitude-encoded information to time. This is beneficial in terms of accuracy and robustness, when the medium that transports or processes the signal (Shannon: 'channel') has a higher capacity in time than in amplitude. VCOs, providing FM, FSK PSK are apparent examples. A sub-class of TEs provides digital output amplitudes (quantized), using a form of pulse-modulation (PM) $[1,2]$. Many of these are combined with a time-todigital converter (TDC) to achieve a discrete-time output. Obvious examples here are all conventional (multi) slope and synchronous SDM ADCs. Advanced versions refine the TDC e.g. with interpolation and calibration [3]; or put it inside an SDM, thus providing noise-shaping on its quantization errors $[3,4,5,6,7,8]$.

A minority of TE converters, however, leaves the time discretization step; indeed, synchronization is only needed when you store the information in memory (time information decoupled), or process it with a fixed time reference: a synchronous clock. From a Shannon standpoint of view [10, 11], time synchronization is superfluous as long as the capacity (and thus accuracy and robustness) of the channel is high enough in the time domain. For silicon, this applies, as technology provides ongoing improvements in time resolution. Asynchronous TEs are used e.g. in switching power converters and power amplifiers (PA) $[1,12,13]$, and, alternatively within an asynchronous SDM (ASDM), see Fig.

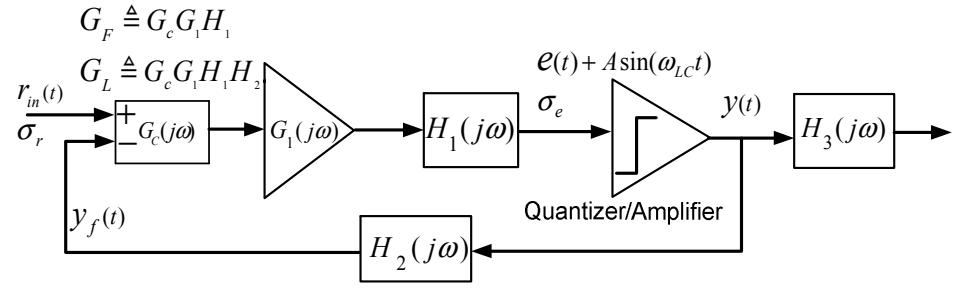

Figure (1): Block Diagram of an ASDM

$1[5]$. Here, AD conversion is followed further in the chain by a filter once spectral selectivity is required.

For these systems, there is an important second advantage of using a TE, not indicated by Shannon: power efficiency and power control (over a wide dynamic range). Indeed, switching is very power-efficient, and important especially for battery-supplied or battery-less applications for mobile, wireless sensor networks, and medical applications.

Leaving the time synchronization leads to a third advantage: the signal does not have the in-band distortion ('noise') due to quantization. Instead, the distortion can be kept out-of-band, and completely suppressed in the post filter. Error-less reconstruction comes into reach, while still using all the advantages of robustness, linearity and accuracy of the TE. This can be traded off for lower power dissipation.

As a fourth advantage, saving sampling circuitry reduces area and power dissipation, and noise and error sources. Clearly, an asynchronous TE data converter, and especially an ASDM, is very promising. However, its analysis is quite complex and its synthesis even more. Current methods lack insight, are too time consuming, do not address the correct metrics, and do not take into account input signal statistics.

This paper provides insight and effective and efficient analysis and synthesis tools for ASDM, for a broader range of metrics, including power efficiency and spectral distribution, appropriate to a wider range of applications than mere data conversion. The results can be extended to synchronous data converters, to provide appropriate specifications for communication-like applications, where signal statistics are not yet, and spectral specifications not efficiently, addressed by the conventional methods. 


\section{EARLIER APPROACHES AND LIMITATIONS}

It is obvious that the design process of any TE convertor is a compromise of speed, SNR and power consumption $\left(P_{c o n}\right)$. Analytic expression of these key performance metrics versus design parameters has been a great challenge. While having severe limitations already for SDM, the simple white-noise model, is inappropriate for ASDM. Numerical modeling techniques (transient, harmonic balance, etc.) are very time consuming and, lack insight. Methods based on multipleinput describing functions are only valid for a very restricted set of input signals, and do not address issues like peak-toaverage ratio (PAR), linearity, spectral purity and errorvector-magnitude (EVM). In [5], an extended DF-based model is elaborated to describe ASDM (and SDM), showing very useful new insights in limit-cycle behavior, but still lacking a relation to input statistics. Mladenov specifically addressed stability analysis [14]. In [2], it has been assumed that a closed form expression for output waveform exists. This assumption falls short in predicting in-band nonlinearities (and thus EVM, etc), especially when the structure is adopted as a transmitter [17]. [12] proposed methods to predict nonlinear metrics and bandwidth of this structure for simplistic situations like sinusoidal inputs and expresses the third order distortion as a function of sinusoidal input for a specific implementation of SDM. The approach presented in [16], introduces a comprehensive method which could be used to analyze the convertor SNR for a general random Gaussian input. It uses describing functions to extract the input of the quantizer, versus the input of the loop, which could subsequently be used to calculate the output spectrum and nonlinear metrics, like EVM.

\section{ANALYSIS METHOD}

The approach proposed in this paper for nonlinear analysis provides analytical expressions and huge calculation speed up, for the three basic metrics for an ASDM: distortion, bandwidth and power consumption, which can be used to aid the tradeoffs at design. It develops the idea to address noise more in line with Shannon as (in-band and out-band) uncorrelated nonlinear distortion at the quantizer output, for given input-signal statistics. Also the power definition is elaborated, to make it valid for PA applications too, where the quantizer (PA) is the dominant block with respect to power efficiency, whereas it can be neglected in normal data converters.

Based on Gaussianality of the input signal and series expansion of the output autocorrelation with Hermite polynomials, a new set of coefficients is presented which could be used to extract the spectral metrics of the output, with the advantage of giving the uncorrelated output distortion (nonlinear noise) and output linear components, from the same set of calculations. The coefficients are described in equations (1) and (2); and (3) denotes the output autocorrelation [16].

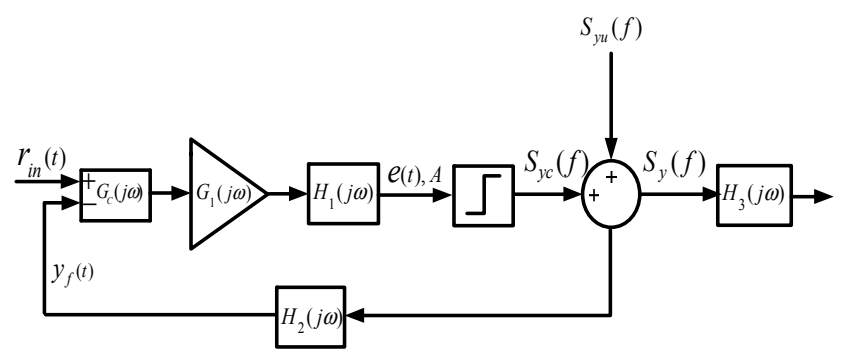

Figure (2): Output uncorrelated part is processed by the loop

$$
\begin{aligned}
& c_{k}\left(A, \sigma_{e}\right)=\frac{(2 \pi)^{-\frac{3}{2}}}{\sigma_{e}^{k} \sqrt{k !}} \int_{-\infty}^{\infty} e^{-\frac{r^{2}}{2}} H_{k}(r) y_{1}(r) d r \\
& y_{1}(r)=\int_{0}^{2 \pi} y\left(\sigma_{e} r+A \sin (\theta)\right) d \theta \\
& R_{y y}(\tau)=\sum_{k=1}^{\infty} c_{k}^{2} R_{e e}^{k}(\tau)
\end{aligned}
$$

The integration process in equation (2) extracts the static equivalent nonlinearity, which is a linearized version of the hard nonlinearity, while $\theta$ represents the random phase of the limit cycle; then (1) uses the Gaussian distribution of the random output component, to find the auto correlation coefficients. Equation (2) can be written for any kind of dithering signal, like multiple sinusoids or saw-tooth. For special cases like ideal relay quantizer, the solution of integral (1) can be expressed in closed form in terms of Bessel functions.

Series expansion of (3) requires knowledge of the amplifier input signal which can be calculated by simultaneous solution of two different equations: the transfer function from the input of the loop to the input of the amplifier, and the oscillation condition at limit cycle frequency (round trip gain of one). These two conditions yield to two equations versus A and $\sigma$ which enable us to calculate them [17].

$$
\begin{aligned}
& S_{y}(\omega)=S_{y u}(\omega)+S_{y c}(\omega) \\
& S_{y u}(\omega)=\mathcal{F}\left(\sum_{k=3}^{\infty} c_{k f}^{2} R_{e e}^{k}(\tau)\right) \\
& c_{k f}=c_{k}\left|\frac{1}{1+G_{L} \cdot N_{r}(A, \sigma)}\right|^{2}
\end{aligned}
$$

Equations (4-a,b,c) represent the output spectrum decomposed to two explicitly separate spectral densities, namely correlated and uncorrelated. The uncorrelated part can be treated as a nonlinear noise, related to the input signal amplitude that affects SNR, and equation (4-c) represents the effect of the feedback loop on linearization, assuming that the nonlinear intermodulations are small enough. As depicted in Fig. (2) the uncorrelated distortion will be further processed by the loop and the total nonlinear in-band distortion power can be calculated by integration of equation (4-b) and splitting the in-band part of the power, $N_{d}$, in equation (5$\mathrm{a}, \mathrm{b})$.

$$
\begin{gathered}
N_{d}=\sum_{k=3}^{\infty} \beta(k) c_{k f}^{2} \int_{<B W>}\left|\frac{S_{r}(\omega) G_{F}(\omega)}{1+G_{L}(\omega) N_{r}\left(A, \sigma_{e}\right)}\right|^{2} d \omega \\
\beta(k)=\frac{1}{2^{k-1}}\left(\begin{array}{c}
k \\
\frac{k-1}{2}
\end{array}\right),
\end{gathered}
$$



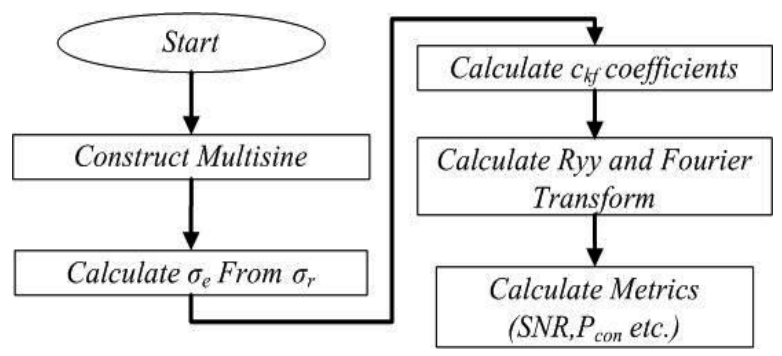

Figure (3): Flow chart of the presented analysis approach

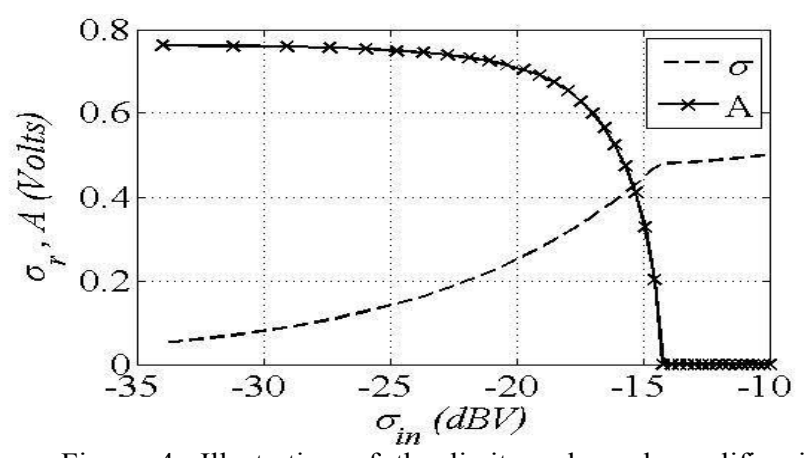

Figure 4: Illustration of the limit cycle and amplifier input amplitudes versus loop input amplitude for a sample loop

while $\beta(k)$ is the ratio of the in-band uncorrelated distortion part to the total distortion. Fig. (3) sums up the suggested method, as an ordered procedure. The input can be approximated with a multisine that mimics the statistics of the input signal $[17,18]$. Fig. (4) shows the trend of limit cycle and random component variations versus loop input rms value. The decrease in limit cycle amplitude deteriorates the nonlinearity. As a result, SNR is readily given as the ratio between the correlated and uncorrelated output powers:

$$
S N R=\frac{c_{1}^{2} \sigma_{e}^{2}}{N_{d}}
$$

Continuation of oscillation puts an upper limit for the input range, i.e. for equal limit cycle and input rms value we can write:

$$
\sigma_{i n, \max }=D \sqrt{\frac{1}{2 \pi}}\left|H_{2}\left(\omega_{r}\right)+\frac{\alpha\left(\omega_{L C}\right)}{G_{F}\left(\omega_{r}\right)}\right|
$$

where $\mathrm{D}$ is the quantizer step and alpha is a constant. The system bandwidth for a band pass signal around a frequency $f_{r}$ can be derived from the conditions to avoid overlap between the limit cycle even order spurious and input spectrum as illustrated in Fig. (5).

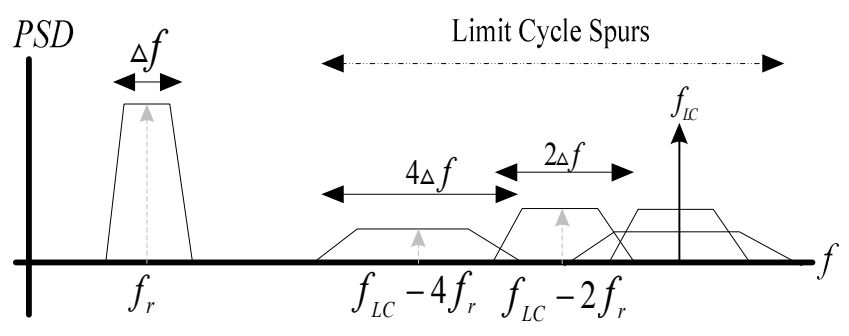

Figure (5): Spectral Content of the TDE output
Eq. (8) describes the maximum instantaneous bandwidth and maximum carrier frequency, based on Fig. (5):

$\Delta f_{\max }=\frac{2}{3} f_{L C}-2 f_{r}, f_{r}<\frac{f_{L C}}{3}$

The power consumption for the convertor is the sum of all consumptions of all blocks and is given by:

$$
P_{\text {con }}=P_{\text {static }}+P_{\text {quantizer }}
$$

The two terms refer to static loss and inverter loss. For transmitter applications, power efficiency is of prime concern. A closed form formula for power consumption (and power efficiency) of a limit cycle power amplifier, relating power consumption/efficiency to $\sigma$ and $A$ is given in [19]

$$
P_{\text {con }}=\frac{g_{0} D^{2}}{2}\left[1-\sum_{m, n=1}^{\infty} G_{m n}(A, \sigma) \operatorname{Real}\left(\frac{g_{0}}{g_{0}+Y_{L}\left(m \omega_{r}+n \omega_{L C}\right)}\right)\right]
$$

where $\mathrm{g}_{0}$ is the switch conductance, $Y_{L}$ is the load admittance, $m, n$ are cross modulation indexes and $G_{m n}$ are gains of cross modulated components of $\omega_{r}$ and $\omega_{L C}$ versus input.

\section{SYNTHESIS METHOD}

The synthesis procedure implies an optimal selection of design parameters to get a predefined set of performance metrics for the amplifier system. Filters $\mathrm{H}_{2}$ and $\mathrm{H}_{3}$ are selected for oscillation with minimum attenuation and proper sharpness for extraction of the desired signal respectively. The important design parameters that can affect the system performance metrics are: 1) forward loop gain for signal and limit cycle, 2) oscillation frequency and 3) quantizer step. The introduced analysis method can be adopted to attain a synthesis insight for optimum power consumption-SNR trade off versus input dynamic range and oscillation frequency, for a given quantizer. The dynamic range is directly proportional to the quantizer step as expressed in equation (7).

Fig. (6-a) illustrates the output signal power and distortion power trade-off versus the input signal rms value. Transmitter applications often require a certain distortion level at the output that for a given loop determines the input dynamic range for standard compliance, and corresponding output power. The trade-off between the minimum SNR (at maximum input) and the maximum input dynamic range, versus forward signal gain is illustrated in Fig. (6-b). The dynamic range decreases monotonically versus the forward gain but the linearity deteriorates to some point for gain and improves afterwards with increasing gain. The reason is that increasing gain, initially increases the input signal to the quantizer, but linearizes the TDE after the minimum point. Therefore forward gain is a useful degree of freedom for linearization.

Another important trade off in converter synthesis is between SNR and power dissipation (or linearity-efficiency trade-off) versus input rms value and is illustrated in (6-c). Bandwidth and power consumption are compromised, as plotted versus oscillation frequency plotted in Fig. (6-d). Using the formulas given in (6) to (10) a design flow can be developed to optimize SNR, power consumption/efficiency. 

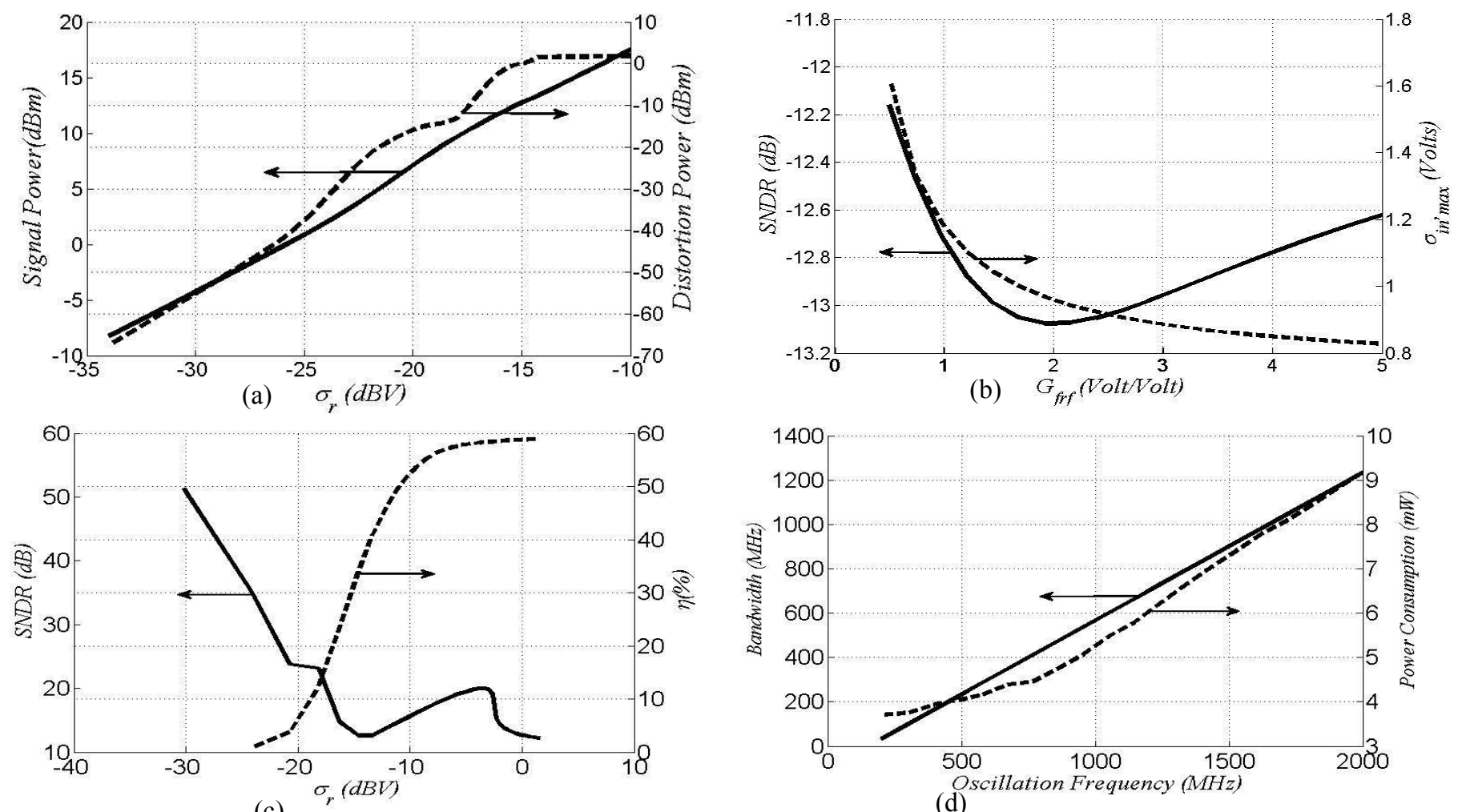

(d)

Figure 6: Typical performance metrics and trade offs for a $3^{\text {rd }}$ order loop with $G_{f r}=2.5, D=1.5$ Volt $, G_{f L C}=1,\left|H_{2}\left(f_{r}\right)\right|=1\left|H_{2}\left(f_{L C}\right)\right|=$ 0.8, (a): output signal and distortion power versus loop input rms; (b): maximum input value versus forward signal gain and SNDR at the maximum input (maximum SNDR); (c): SNDR and efficiency for a typical Class D amplifier in TDE versus rms input; (d): bandwidth and power consumption of the modulator versus limit cycle frequency

\section{CONCLUSION}

By translating the strongly-non-linear quantizer function into a weakly-non-linear one and applying Hermite expansion in the autocorrelation domain, a new, universal fast and efficient analysis and synthesis method is obtained for asynchronous time encoders with optimized spectral metrics, for random inputs. This enables both greatly improved power and channel efficiency, for an extended range of applications and it can be extended to synchronous systems.

\section{REFERENCES}

[1] Srinath Ramaswamy et. al., "A High-Performance Digital-Input ClassDAmplifier with Direct Battery Connection in a 90nmDigital CMOS Process"; ISSCC 2008.

[2] E. Roza, "Analog-to-digital conversion via duty-cycle modulation", IEEE TCAS II, August 1997.

[3] V. Dhanasekaran et.al.,"A 20MHz BW 68dB DR CT $\Delta \Sigma$ ADC based on a multi-bit time-domain quantizer and feedback element"; ISSCC 2009.

[4] Daniels, J. et. al.; "A/D conversion using an Asynchronous DeltaSigma Modulator and a time-to-digital converter", ISCAS 2008.

[5] Ouzounov et. al., "Design of high-performance asynchronous sigma delta modulators with a binary quantizer with hysteresis“, CICC, pp.181-184, October 2004.

[6] Min Park et. al. "A VCO-based Analog-to-Digital Converter with Second-Order Sigma Delta Noise Shaping", ISCAS 2009.

[7] Prefasi et.al; "Analog-to-Digital Conversion Using Noise Shaping and Time Encoding”; TCAS I, August 2008.
[8] Colodro, F.;et. al; "Continuous-Time Sigma-Delta Modulator With an Embedded Pulsewidth Modulation", IEEE TCAS I, April 2008.

[9] Straayer, M.Z et. al; "A Multi-Path Gated Ring Oscillator TDC With First-Order Noise Shaping” JSSC, April 2009.

[10] A.H.M van Roermund "An integral shannon-based view on smart front-ends";EuWiT 2008.

[11] A.H.M van Roermund , "Smart Frond-Ends, from Vision to Design" IEICE Trans. Electron., Vol.E92-C, No.6, June 2009.

[12] T. Piessens. M. Steyaert, "Behavioral analysis of self-oscillating class D line drivers", IEEE TCAS I, April 2005.

[13] Arthur Gelb et. al, Multiple-Input Describing Functions and Nonlinear System Design, New York: Mc-Graw Hill.

[14] Valeri Mladenov, "On the stability of high order Sigma-Delta modulators"; ICECS 2001.

[15] F.Arfaei Malekzadeh et. al" A new approach for nonlinear metric estimation of limit cycle amplifiers", EUMC, Sepmtember 2009.

[16] F. Arfaei Malekzadeh et. al, "A new statistical approach for nonlinear analysis of limit cycle amplifiers", IRWS, January 2009.

[17] K. A. Remley "Multisine excitation for ACPR measurements" IMS 2003.

[18] Kevin G. Gard et.al. "Characterization of Spectral Regrowth in Microwave Amplifiers Based on the Nonlinear Transformation of a Complex Gaussian Process", IEEE MTTS, JULY 1999.

[19] M. Sarkeshi et.al," Efficiency Analysis of aLimit-Cycle Class-D Amplifier With a Random Gaussian Excitation”, IMS 2009. 